\title{
DIFFERENT SAMPLE TYPES IN PIGS CHALLENGED WITH HAEMOPHILUS PARASUIS FOLLOWING TWO TREATMENT SCHEMES WITH TULATHROMYCIN
}

\author{
Andreas PALZER ${ }^{1 *}$, Rose-Leah Austin-Busse ${ }^{1}$, Andrea LAdinIG ${ }^{2}$, Gyula BALKA $^{3}$, \\ Joachim SPERGSER ${ }^{4}$ and Mathias RITZMANN ${ }^{1,2}$ \\ ${ }^{1}$ Clinic for Swine, Ludwig-Maximilians-University Munich, Sonnenstrasse 16, D-85764 \\ Oberschleissheim, Germany; ${ }^{2}$ Clinic for Swine, Department for Farm Animals and \\ Veterinary Public Health and ${ }^{4}$ Institute of Bacteriology, Mycology and Hygiene, \\ Department for Pathobiology, University of Veterinary Medicine, Vienna, Austria; \\ ${ }^{3}$ Department of Pathology and Forensic Veterinary Medicine, Faculty of Veterinary \\ Science, Szent István University, Budapest, Hungary
}

(Received 4 July 2014; accepted 17 February 2015)

This study aimed to test the efficacy of samplings for the detection of Haemophilus parasuis after metaphylactic treatment and subsequent challenge using an established model for Glässer's disease. In this model, 36 piglets were equally assigned to a negative control, a positive control, and two trial groups receiving tulathromycin 7 or 4 days prior to challenge. The piglets of three groups were challenged intratracheally with $H$. parasuis serovar 5. As a result, four pigs in each challenged group died or had to be euthanised within 10 days post challenge. The remaining 15 pigs of these challenged groups survived until termination of the experiment (days 14-15). All pigs were necropsied and collective swabs of serosal surfaces were tested by bacterial culture and PCR. Samples of tarsal synovial fluid and joint capsule, cerebrospinal fluid (CSF) and brain swabs were tested by PCR. A total of 22 out of the 27 challenged animals had macroscopically detectable polyserositis and all of them tested positive in the collective swab samples. Haemophilus parasuis was more frequently detected in pigs that died within the first 10 days compared to those surviving until days 14-15 $(\mathrm{P}<$ 0.001 ), and those that succumbed within 10 days showed higher positivity rates in the brain and CSF. All pigs which were positive in the CSF had detectable meningitis. At days 14-15, joint samples from 5 of the remaining 15 pigs tested positive for $H$. parasuis. Four of these five animals did not show any macroscopic or histological lesions in the joints. In conclusion, collective swabs were the best sample material in acute cases, whereas samples from the joints gave the best results in chronic cases. In this challenge model it was not possible to prove the metaphylactic effect of tulathromycin administered 4 and 7 days prior to infection with $H$. parasuis.

Key words: Experimental infection, Haemophilus parasuis, PCR, swab samples

*Corresponding author; E-mail: a.palzer@Imu.de; Phone: 0049 (89) 2180-78900;

Fax: 0049 (89) 2180-78902 
The aims of this study were to test the efficacy of samplings for the detection of Haemophilus parasuis after metaphylactic treatment and to test the metaphylactic efficacy of tulathromycin against Glässer's disease in an established challenge model. Tulathromycin is used for the therapeutic and metaphylactic treatment of respiratory diseases in pigs (Nanjiani et al., 2005; Callens et al., 2012). Blanco et al. (2004) evaluated disease development in sow-reared (SR) and colostrum-deprived (CD) piglets after intratracheal inoculation with $10^{6}$ to $10^{9}$ colony-forming units (cfu)/ml of $H$. parasuis serovar 5 . More severe signs were observed in the $\mathrm{CD}$ pigs which developed typical symptoms and lesions of Glässer's disease compared to SR pigs. Oliveira et al. (2003) confirmed the suitability of naturally farrowed, colostrum-deprived piglets as a model to study $H$. parasuis infections. The advantage of that model is the easy availability of animals even from herds which are $H$. parasuis positive. However, the survival rate of piglets depends on strict hygiene regimes and the use of antibiotics. Although the survival rate of these pigs has reached $80 \%$, high pre-challenge death losses still occur (Oliveira et al., 2003). Varying inoculation techniques are used in experimental studies. In most studies an intranasal challenge technique is used to infect pigs with $H$. parasuis (Vahle et al., 1997; Brockmeier et al., 2013; CostaHurtado et al., 2013). Alternative methods are based on intraperitoneal (De la Fuente et al., 2010) or intratracheal infection (Bak and Riising, 2002). The $H$. parasuis serovar 5 used in the present study is one of the most prevalent serovars in samples from pigs with Glässer's disease (Zhang et al., 2012; Luppi et al., 2013).

A preliminary diagnosis of Glässer's disease is based on clinical signs and the presence of typical lesions such as polyserositis and polyarthritis. Although a definitive diagnosis of infection typically requires isolation of the causal agent from these lesions, confirmation of an agent by PCR testing is becoming increasingly valuable, especially when the agent is difficult to isolate in pure culture (Palzer et al., 2006; Palzer et al., 2008). Ideally, sites such as the brain (meninges), the pericardium, the pleura, the peritoneum, and the joints should be used (Oliveira, 2004). Various authors have isolated $H$. parasuis from pleural fluid, peritoneal fibrin and pericardial fluid (Neil et al., 1969; Amano et al., 1997; Turni and Blackall, 2007). The same authors have found that the lungs, heart, heart blood, affected joints and brain are the best sites for sampling when an acute infection is present. Interestingly, it has been described that pigs infected with virulent isolates of $H$. parasuis can remain healthy and serve as reservoirs for transmission to naïve pigs (Brockmeier et al., 2013). However, no reports are available about the optimal sampling system for the detection of $H$. parasuis at different times after metaphylactic treatments and challenge. The objectives of this study were to test the efficacy of a metaphylactic treatment with tulathromycin either 4 or 7 days prior to experimental infection with $H$. parasuis serovar 5 and to compare different sample types for the diagnosis of Glässer's disease. 


\section{Materials and methods}

\section{Animal experiments}

Thirty-six castrated male crossbred (Landrace $\times$ Large White $\times$ Pietrain) piglets were obtained at three weeks of age from a commercial farrowing farm with no prior history of porcine reproductive and respiratory syndrome virus (PRRSV) infection or Glässer's disease in the sow herd. The piglets had been randomly selected from a total of 18 litters and had been bled at one week of age, together with their dams; all samples tested negative for antibodies against $H$. parasuis by ELISA (INGEZIM ${ }^{\circledR}$ Haemophilus 11.HPS.K 1, Ingenasa, Madrid, Spain). Piglets were vaccinated against Mycoplasma hyopneumoniae (Hyoresp ${ }^{\circledR}$, Merial, Lyon, France) at one and three weeks of age and against PCV2 (Porcilis ${ }^{\circledR}$ PCV, MSD, Boxmeer, The Netherlands) at three weeks of age. Piglets were moved to the Clinic for Swine (University of Veterinary Medicine Vienna, Austria) and allowed to acclimatise for a period of 17 days prior to challenge. The study was conducted over a 32-day period which included a 14- to 15-day postchallenge evaluation. On arrival, piglets were weighed and assigned to one of four treatment groups (Groups 1-4) according to their weight in order to establish a uniform group weight distribution.

The study was originally designed to evaluate the efficacy of tulathromycin by treating weaned piglets according to two different protocols. Piglets received a tulathromycin treatment at a dosage of $2.5 \mathrm{mg}$ per $\mathrm{kg}$ body weight (BW) at either seven or four days prior to infection with a virulent $H$. parasuis serotype 5 . Pigs from Groups 3 and 4 received an intramuscular injection with tulathromycin on days -7 and -4 . The minimum inhibitory concentration for the $H$. parasuis strain used was $0.5 \mathrm{mg} / \mathrm{l}$. To prepare the inoculum, the strain was streaked onto a chocolate agar, which was incubated for 4 days at $37^{\circ} \mathrm{C}$ in an atmosphere of $7 \%$ carbon dioxide. Colonies were harvested in phosphate-buffered saline (PBS). The cfu count in one aliquot was determined using serial dilution and plating (Viehmann et al., 2013), and aliquots were kept frozen $\left(-80^{\circ} \mathrm{C}\right)$ until further use. One day prior to challenge, on Day -1 , one aliquot was selected, thawed at $37^{\circ} \mathrm{C}$ and the suspension was adjusted to the colony count of approximately $1 \times 10^{8} \mathrm{cfu} / \mathrm{ml}$. On the day of challenge (day 0), pigs from Groups 2, 3 and 4 were inoculated intratracheally with $5 \mathrm{ml}$ of $H$. parasuis strain Nagasaki (serotype 5) containing $10^{8} \mathrm{cfu} / \mathrm{ml}$. The sensitivity of the strain to tulathromycin was tested in vitro. Pigs from the control group were mock infected using PBS. After challenge, pigs were examined at least once daily until study termination. The following clinical parameters were included in the examination: body temperature, condition, respiratory signs like coughing, filling of the joints, appearance of the conjunctiva and sclera, behaviour and signs of pain, abdominal appearance and the backline of the pigs, lameness and central nervous system (CNS) symptoms. Animals that showed severe clinical signs, such as recumbency with inability to rise, were 
euthanised. Those animals were sedated and then euthanised with T61 ${ }^{\circledR}$ (Intervet, Boxmeer, The Netherlands) via intracardiac injection. At termination, the remaining animals were euthanised in the same way. All pigs were necropsied directly after death or euthanasia, and the gross pathological findings were recorded.

Animal care and euthanasia during the study were approved and conducted in accordance to the current Animal Experiments Act and in accordance with the Good Scientific Practice guidelines of the Ethics Commission of the Veterinary University Vienna, Austria. The study was registered by the Austrian Federal Ministry for Science and Research, Department for Genetics and Animal Experiments/Testing.

\section{Detection of $\mathrm{H}$. parasuis}

Collective swabs (sterile cotton swabs embedded in Amies charcoal transport medium; Medi Swab ${ }^{\circledR}$, Heinz Herenz, Hamburg, Germany) from serosal surfaces (pleura, pericardium, peritoneum) were taken from all pigs for bacterial culture and PCR to detect $H$. parasuis (Palzer et al., 2006). In addition, samples for PCR were collected from the synovial fluid and joint capsule from both tarsal joints and any other swollen joints as well as from the cerebrospinal fluid (CSF) and brain (meningeal swabs). CSF was collected from either the occipital or the lumbosacral region. DNA was extracted from the samples using the GenElute ${ }^{\circledR}$ Mammalian Genomic DNA Miniprep Kit (Sigma-Aldrich Biotechnology) according to the manufacturer's user guide. Approximately $250 \mu \mathrm{L}$ of undiluted DNA was obtained after extraction and $5 \mu \mathrm{L}$ of DNA were used for PCR analysis. PCR testing according to the method described by Angen et al. (2007) and bacterial culture were performed at the Institute of Bacteriology, Mycology and Hygiene, University of Veterinary Medicine Vienna, Austria. Bacterial culture on chocolate blood agar was started within $30 \mathrm{~min}$ of the collection of serosal swabs. The cultures were incubated at $37{ }^{\circ} \mathrm{C}$ for $2-3$ days in a $7 \% \mathrm{CO}_{2}$ aerobic environment. Suspect colonies were subcultured onto a new chocolate blood agar plate and incubated for 3-4 days. Colonies were confirmed as H. parasuis by PCR.

\section{Statistical analysis}

Statistical analysis was performed using SPSS 17.0 for Windows XP. Descriptive statistics were done using either SPSS 17.0 or Microsoft Office Excel 2007. The Kaplan-Meier test was used to calculate overall mortalities between the individual groups, while all other group comparisons were analysed using the Mann-Whitney U test. Haemophilus parasuis detection methods were compared using the Kappa test. In the analyses, group comparisons were performed between Groups 1 and 2, Groups 2 and 3, Groups 2 and 4, and finally Groups 3 and 4. 
The $1.25 \%$ level of significance obtained by Bonferroni correction was used to eliminate error for all tests with the exception of the association between detection frequencies of $H$. parasuis where a 5\% significance level was used.

\section{Results}

A total of 12 pigs (four from each challenge group) had to be removed due to death or euthanasia within 10 days of challenge. Two of these pigs were found dead in their pens and the remaining 10 were euthanised due to severe disease with lateral recumbency and inability to rise. None of the control animals were euthanised or died before the end of the study on day 15. Four pigs from each of Groups 2 and 3, and one pig from Group 4 were removed between days 2 and 4 post infection, while the remaining three severely affected pigs in Group 4 were either found dead on day 6 (one pig) or required euthanasia on day 10 (two pigs). There was no significant difference in the time of death among the three challenge groups $(\mathrm{P}>0.05)$.

All samples from the control group tested negative by PCR for H. parasuis, and all serosal swabs from this group were negative by bacterial culture. A total of 129 samples collected from the three challenge groups were examined by PCR. Six out of the 135 possible samples were unsuitable for testing. Culturepositive samples from collective serosal swabs were obtained from 10 out of 27 challenged pigs, all of which suffered from severe disease and were necropsied within 10 days after challenge (Table 1). Haemophilus parasuis was detected by PCR from collective serosal swabs of all the 10 culture-positive animals, as well as from serosal swabs from two additional challenged pigs which were necropsied within the first 10 days post challenge (Table 1). After challenge, detection of $H$. parasuis by culture and PCR was significantly more frequent $(\mathrm{P}<0.001$ for each) in the 12 pigs removed due to disease in the first 10 days than in those 15 which survived for 14-15 days. Overall, $H$. parasuis was detected by PCR in 18 out of 27 challenged pigs (Table 1) with a similar number detected among Groups 2, 3 and 4 ( $\mathrm{n}=7,6$ and 5, respectively; Table 2). PCR detection of $H$. parasuis was most frequent in collective serosal swabs (12 samples) and CSF (nine samples) compared to joint fluid (four samples), joint capsule (five samples) or brain swabs (four samples). In five pigs that survived until termination (days 14-15), H. parasuis specific genome was detected by PCR; joint samples tested positive in all five pigs and CSF in two pigs. All PCR-positive joints were tarsal joints.

A total of 22 animals had macroscopically detectable polyserositis. Six were from Group 2, nine from Group 3, and seven from Group 4. No significant difference was seen between the three challenged groups $(\mathrm{P}>0.0125)$. One animal from Group 1 had pericarditis at necropsy. The same animal had synovitis in 
one tarsal joint. All animals testing positive in the collective swab had polyserositis at necropsy. Synovitis was found in 13 animals of the three challenged groups: four animals from Group 2, six from Group 3 and three from Group 4 $(\mathrm{P}>0.0125)$. Comparing PCR results obtained from macroscopic lesions, only four animals positive in at least one joint sample had synovitis. Five animals were positive by PCR without any lesions; four of them survived until termination and were PCR positive from joint fluid only. Meningitis was seen in 22 animals: six from Group 2, nine from Group 3 and seven from Group 4 (P > 0.0125). All PCR-positive pigs had meningitis at necropsy.

Table 1

Results for 17 out of 27 challenged animals that tested positive in at least one sample by PCR or culture, arranged by the day of death or euthanasia post challenge

\begin{tabular}{|c|c|c|c|c|c|c|c|c|}
\hline \multirow{2}{*}{$\begin{array}{l}\text { Ear tag } \\
\text { number }\end{array}$} & \multirow{2}{*}{ Group } & \multirow{2}{*}{$\begin{array}{l}\text { Days } \\
\text { p. i. }\end{array}$} & \multicolumn{2}{|c|}{ Serosal swabs } & $\begin{array}{c}\text { Joint } \\
\text { capsule }\end{array}$ & $\begin{array}{l}\text { Synovial } \\
\text { fluid }\end{array}$ & $\begin{array}{l}\text { Cerebrospi- } \\
\text { nal fluid }\end{array}$ & $\begin{array}{l}\text { Brain } \\
\text { swab }\end{array}$ \\
\hline & & & Culture & PCR & \multicolumn{4}{|c|}{ PCR } \\
\hline 193 & 2 & 2 & + & + & + & - & - & - \\
\hline 296 & 3 & 2 & + & + & - & - & + & + \\
\hline 298 & 3 & 2 & + & + & + & - & + & + \\
\hline 199 & 2 & 3 & + & + & - & - & + & - \\
\hline 295 & 3 & 3 & + & + & - & - & - & - \\
\hline 496 & 4 & 3 & + & + & - & - & + & - \\
\hline 195 & 2 & 4 & + & + & - & - & - & - \\
\hline 196 & 2 & 4 & + & + & - & - & + & - \\
\hline 293 & 3 & 4 & + & + & - & - & + & + \\
\hline 498 & 4 & 6 & + & + & + & - & + & + \\
\hline 492 & 4 & 10 & - & + & - & - & - & - \\
\hline 494 & 4 & 10 & - & + & + & - & - & - \\
\hline \multicolumn{3}{|c|}{$\begin{array}{l}\text { Number of positive results } \\
\text { before termination }(37 / 72)\end{array}$} & 10 & 12 & 4 & 0 & 7 & 4 \\
\hline 197 & 2 & 14 & - & - & - & + & - & - \\
\hline 291 & 3 & 14 & - & - & - & + & - & - \\
\hline 292 & 3 & 14 & - & - & - & + & - & - \\
\hline 493 & 4 & 14 & - & - & - & + & - & - \\
\hline 194 & 2 & 15 & - & - & + & - & + & - \\
\hline 192 & 2 & 15 & - & - & - & - & + & - \\
\hline \multicolumn{3}{|c|}{$\begin{array}{l}\text { Termination number of } \\
\text { positive results }(7 / 36)\end{array}$} & 0 & 0 & 1 & 4 & 2 & 0 \\
\hline \multicolumn{3}{|c|}{$\begin{array}{l}\text { Total number of positive } \\
\text { results: } n=44\end{array}$} & 10 & 12 & 5 & 4 & 9 & 4 \\
\hline
\end{tabular}


Table 2

PCR results for Haemophilus parasuis from five sites according to treatment group, presented as the number of positive samples

\begin{tabular}{|c|c|c|c|c|c|}
\hline Sample & $\begin{array}{c}\text { Joint } \\
\text { capsule }\end{array}$ & $\begin{array}{l}\text { Synovial } \\
\text { fluid }\end{array}$ & $\begin{array}{l}\text { Cerebrospinal } \\
\text { fluid }\end{array}$ & $\begin{array}{l}\text { Brain } \\
\text { swab }\end{array}$ & $\begin{array}{c}\text { Collective } \\
\text { serosal } \\
\text { swab }\end{array}$ \\
\hline Control group (Group 1), $n=9$ & 0 & 0 & 0 & 0 & 0 \\
\hline Untreated-challenged (Group 2), $n=9$ & 2 & 1 & 4 & 0 & 4 \\
\hline Treated, -4 days (Group 3), $\mathrm{n}=9$ & 1 & 2 & 3 & 3 & 4 \\
\hline Treated, -7 days (Group 4), $n=9$ & 2 & 1 & 2 & 1 & 4 \\
\hline Total number of positive results & 5 & 4 & 9 & 4 & 12 \\
\hline
\end{tabular}

\section{Discussion}

The $H$. parasuis challenge model used in this study induced acute and severe clinical signs which resulted in euthanasia or death of four out of nine pigs from each challenge group within ten days after infection. The high number of deaths is in agreement with findings in previous studies which used highly pathogenic strains of $H$. parasuis serotype 5. According to Aragon et al. (2012), serovar 5 is highly virulent and capable of causing death in pigs. Similarly, other authors reported the deaths of several pigs shortly after experimental infection with $H$. parasuis serovar 5 (Amano et al., 1997; Bak and Riising, 2002; De la Fuente et al., 2010).

In the present study, we found no significant differences in clinical outcome between the three challenged groups. When an organism is inoculated intratracheally, it circumvents the mucosal immunity of the upper respiratory tract and can cause systemic infection more easily (Amano et al., 1997). Therefore, it might be necessary to reduce the dose of the inoculum if an intratracheal challenge model is used to induce the disease in order to see the effect of an antimicrobial treatment. Further studies are necessary to determine appropriate inoculum dosage and tulathromycin administration time in this challenge model. Another issue may be the time-points chosen for treatment. These time-points were chosen to cover the half-life of tulathromycin which is 3.75 days (Group 3) and twice the half-life (Group 4) (Benchaoui et al., 2004). Tulathromycin is registered in the European Union for the therapeutic and metaphylactic treatment of pneumonia caused by $H$. parasuis. The time-points were chosen to simulate the different contact situations in a group treatment of pigs. Furthermore, the concentrations of tulathromycin in the lungs are higher than in the plasma but are not detectable after six days (Yang et al., 2013). Therefore, the concentration of tulathromycin in the lungs at the moment of the challenge was possibly insufficient to prevent disease in our model. As a consequence, in this challenge model it was 
not possible to prove the metaphylactic effect of tulathromycin administered 4 and 7 days prior to infection with $H$. parasuis.

Haemophilus parasuis DNA could be detected by bacterial culture in samples from 18 challenged pigs. Haemophilus parasuis was detected in at least one of five samples from pigs of all three challenged groups by PCR and was isolated in 10 collective serosal swabs from pigs of all three challenged groups. As expected, H. parasuis DNA was not detected by PCR or culture in samples from the control pigs. Although a significant difference among the sample types for the detection of $H$. parasuis by PCR was not established in this study, the bacterium was most frequently detected in collective serosal swabs and cerebrospinal fluids. This detection frequency corresponds to findings in other studies (Palzer et al., 2006). Swabs can be used successfully for sampling diseased animals for H. parasuis diagnosis, particularly by PCR (Angen et al., 2007). In this study, the number of positive samples was higher in the fluid than in the swab of the brain. The highest success rate for $H$. parasuis detection by either PCR or bacterial culture was achieved when a combination of samples from the meninges, peritoneum and pleura was used (Blanco et al., 2008). Likewise, Oliveira and Pijoan (2002) noted that ideal sites for isolation were the brain (meninges), the pericardium, the pleura, the peritoneum and the joints. All these recommended sampling sites were investigated in this study. The investigation of brain swabs produced no additional findings, because all positive animals were positive in the corresponding cerebrospinal fluid, which seems to be a better sample type.

Haemophilus parasuis was most frequently detected by PCR in synovial fluid from animals that were euthanised at the end of the trial. Notably, the bacteria were not concurrently detected by PCR in the corresponding joint capsules of these pigs. When $H$. parasuis was detected in joint capsules of other pigs throughout the study period, the synovial fluid consistently tested negative. Therefore, at no point in this study was the $H$. parasuis genome identified in both the synovial fluid and the joint capsules. Differences in the detection frequency of $H$. parasuis may be due to different amounts of bacteria present in the synovial fluid and joint capsules. For other PCR protocols it is known that a minimum of $100 \mathrm{cfu} / \mathrm{mL}$ is required for detection (Oliveira et al., 2001). The sensitivity of the PCR method used in this study was reported to be slightly lower than that of other PCR methods (Oliveira et al., 2001; Yang et al., 2013). Another explanation might be that the bacterium is first localised in the joint capsule during the early stages of infection, while in later stages it is only present in the synovial fluid. This assumption is supported by the fact that $H$. parasuis was only detected in the synovial fluids of animals that died at study termination. Further investigations are required to understand the mechanism of $H$. parasuis colonisation of the joints.

Although $H$. parasuis was more frequently detected in serosal swabs of challenged pigs by PCR (12 out of 27) than by culture (10 out of 27), and PCR 
detected all cases that were detected by culture, there was no significant difference between the two methods. The very rapid procedure of the bacteriological investigation may have had a positive influence on the number of positive samples. However, in routine diagnostics it is normally not possible to perform a culture within such a short time after necropsy.

Due to severe clinical symptoms 12 pigs from all three challenged groups died or were euthanised within ten days post infection. Haemophilus parasuis was detected in three different sample types (joint capsule, synovial fluid and cerebrospinal fluid) from six pigs which were euthanised at study termination. It is possible that they could have developed more obvious lesions if given more time.

The results of this study confirm that PCR and bacterial examination of various sample types are suitable diagnostic tools to detect all stages of $H$. parasuis infection. In this study, an investigation of collective swabs by PCR gave the best results in acute cases of the disease. In chronic cases the best sample to detect $H$. parasuis was taken from the joints. Regarding samples from the CNS, cerebrospinal fluid was positive in more cases than the corresponding brain swab. All these results indicate that the diagnostic method and sample type must be carefully chosen according to the stage of the disease.

\section{References}

Amano, H., Shibata, M., Takahashi, K. and Sasaki, Y. (1997): Effects on endotoxin pathogenicity in pigs with acute septicemia of Haemophilus parasuis infection. J. Vet. Med. Sci. 59, 451-455.

Angen, O., Oliveira, S., Ahrens, P., Svensmark, B. and Leser, T. (2007): Development of an improved species specific PCR test for detection of Haemophilus parasuis. Vet. Microbiol. 119, 266-276.

Aragon, V., Segalés, J. and Oliveira, S. (2012): Glässer's disease. In: Zimmerman, J., Karriker, L., Ramirez, A., Schwartz, K. and Stevenson, G. (eds) Diseases of Swine. 10th edition. WileyBlackwell, Chichester, UK. pp. 760-778.

Bak, H. and Riising, H. (2002): Protection of vaccinated pigs against experimental infections with homologous and heterologous Haemophilus parasuis. Vet. Rec. 151, 502-505.

Benchaoui, H., Nowakowski, M., Sherington, J., Rowan, T. and Sunderland, S. (2004): Pharmacokinetics and lung tissue concentrations of tulathromycin in swine. J. Vet. Pharmacol. Ther. 27, 203-210.

Blanco, I., Canals, A., Evans, G., Mellencamp, M., Cia, C., Deeb, N., Wang, L. and GalinaPantoja, L. (2008): Differences in susceptibility to Haemophilus parasuis infection in pigs. Can. J. Vet. Res. 72, 228-235.

Blanco, I., Galina-Pantoja, L., Oliveira, S., Pijoan, C., Sanchez, C. and Canals, A. (2004): Comparison between Haemophilus parasuis infection in colostrum-deprived and sow-reared piglets. Vet. Microbiol. 103, 21-27.

Brockmeier, S. L., Loving, C. L., Mullins, M. A., Register, K. B., Nicholson, T. L., Wiseman, B. S., Baker, R. B. and Kehrli, M. E. (2013): Virulence, transmission, and heterologous protection among four isolates of Haemophilus parasuis. Clin. Vacc. Immunol. 20, 1466-1472. 
Callens, B., Persoons, D., Maes, D., Laanen, M., Postma, M., Boyen, F., Haesebrouck, F., Butaye, P., Catry, B. and Dewulf, J. (2012): Prophylactic and metaphylactic antimicrobial use in Belgian fattening pig herds. Prev. Vet. Med. 106, 53-62.

Costa-Hurtado, M., Olvera, A., Martinez-Moliner, V., Galofré-Milà, N., Martínez, P., Dominguez, J. and Aragon, V. (2013): Changes in macrophage phenotype after infection of pigs with Haemophilus parasuis strains with different levels of virulence. Infect. Immun. 81, 2327-2333.

De la Fuente, A., Carpintero, R., Rodriguez Ferri, E., Alava, M., Lampreave, F. and Gutierrez Martin, C. (2010): Acute-phase protein response in pigs experimentally infected with Haemophilus parasuis. Comp. Immunol. Microbiol. Infect. Dis. 33, 455-465.

Luppi, A., Bonilauri, P., Dottori, M., Iodice, G., Gherpelli, Y., Merialdi, G., Maioli, G. and Martelli, P. (2013): Haemophilus parasuis serovars isolated from pathological samples in Northern Italy. Transbound. Emerg. Dis. 60, 140-142.

Nanjiani, I. A., McKelvie, J., Benchaoui, H. A., Godinho, K. S., Sherington, J., Sunderland, S. J., Weatherley, A. J. and Rowan, T. G. (2005): Evaluation of the therapeutic activity of tulathromycin against swine respiratory disease on farms in Europe. Vet. Ther. 6, 203-213.

Neil, D., McKay, K., L'Ecuyer, C. and Corner, A. (1969): Glässer's disease of swine produced by the intratracheal inoculation of Haemophilus suis. Can. J. Comp. Med. 33, 187-193.

Oliveira, S. (2004): Improving rate of success in isolating Haemophilus parasuis from clinical samples. J. Swine Health Prod. 12, 308-309.

Oliveira, S. and Pijoan, C. (2002): Diagnosis of Haemophilus parasuis in affected herds and use of epidemiological data to control disease. J. Swine Health Prod. 10, 221-225.

Oliveira, S., Blackall, P. and Pijoan, C. (2003): Characterization of the diversity of Haemophilus parasuis field isolates by use of serotyping and genotyping. Am. J. Vet. Res. 64, 435-442.

Oliveira, S., Galina, L. and Pijoan, C. (2001): Development of a PCR test to diagnose Haemophilus parasuis infections. J. Vet. Diagn. Invest. 13, 495-501.

Palzer, A., Ritzmann, M., Hafner-Marx, A., Wolf, G. and Heinritzi, K. (2006): Detection of Haemophilus parasuis and Mycoplasma hyorhinis in swine and association of those pathogens with clinical and pathological findings. Dtsch. Tierärztl. Wochenschr. 113, 209-248.

Palzer, A., Ritzmann, M., Wolf, G. and Heinritzi, K. (2008): Association between pathogens in healthy pigs and pigs with pneumonia. Vet. Rec. 162, 267-271.

Turni, C. and Blackall, P. (2007): Comparison of sampling sites and detection methods for Haemophilus parasuis. Aust. Vet. J. 85, 177-184

Vahle, J., Haynes, J. and Andrews, J. (1997): Interaction of Haemophilus parasuis with nasal and tracheal mucosa following intranasal inoculation of cesarean derived colostrum deprived (CDCD) swine. Can. J. Vet. Res. 61, 200-206.

Viehmann, M., Postiasi, S., Balka, G., Spergser, J., Palzer, A., Hennig-Pauka, I., Ritzmann, M. and Ladinig, A. (2013): Evaluation of the efficacy of a combination therapy of an antibiotic and a NSAID following an experimental Haemophilus parasuis infection in nursery piglets. Tierärztl. Prax. 41, 225-232.

Yang, F., Huang, X. H., Li, G. H., Ni, H. J., Zhao, Y. D., Ding, H. Z. and Zeng, Z. L. (2013): Estimating tulathromycin withdrawal time in pigs using a physiologically based pharmacokinetics model. Food Addit. Contam. Part A 30, 1255-1263.

Zhang, J., Xu, C., Guo, L., Shen, H., Deng, X., Ke, C., Ke, B., Zhang, B., Li, A., Ren, T. and Liao, M. (2012): Prevalence and characterization of genotypic diversity of Haemophilus parasuis isolates from southern China. Can. J. Vet. Res. 76, 224-229. 